\title{
Dietary patterns and the incidence of hyperglyacemia in China
}

\author{
Xin Hong ${ }^{1}$, Fei $\mathrm{Xu}^{1,2, *}$, Zhiyong Wang ${ }^{1}$, Yaqiong Liang ${ }^{1}$ and Jiequan $\mathrm{Li}^{1}$ \\ 'Department of Non-communicable Disease Prevention, Nanjing Municipal Center for Disease Control \& Prevention, \\ 2 Zizhulin, Nanjing 210003, People's Republic of China: ${ }^{2}$ Department of Epidemiology and Biostatistics, School of \\ Public Health, Nanjing Medical University, Nanjing, People's Republic of China
}

Submitted 4 August 2014: Final revision received 6 January 2015: Accepted 4 February 2015: First published online 16 April 2015

\begin{abstract}
Objective: Epidemiological studies have examined associations between dietary patterns and the risk of type 2 diabetes. However, information on dietary patterns and the risk of type 2 diabetes in Chinese populations is scarce. The aim of the present study was to identify dietary patterns and examine their association with incident hyperglycaemia in Nanjing, China.

Design: A community-based prospective cohort study. Dietary assessment was carried out using a validated eighty-seven-item FFQ. Dietary patterns were identified by exploratory factor analysis. Participants were categorized into tertiles of dietary factor score for each dietary pattern. The relationship between dietary patterns and hyperglycaemia risk was analysed using multivariable linear and Cox regression.

Setting: Seven communities from two urban districts in Nanjing, China.

Subjects: A total of 2900 of Chinese local residents aged 30 years or above, free of hyperglycaemia and other serious diseases, who participated in the baseline survey from June to September 2007 were followed up 3 years later from June to September 2010 for the development of hyperglycaemia. Fasting blood samples were collected at both baseline and 3-year follow-up surveys. Hyperglycaemia was defined as fasting plasma glucose concentration of $\geq 6 \cdot 1 \mathrm{mmol} / 1$ or already taking oral hyperglycaemia agents for treatment of type 2 diabetes.

Results: Five major dietary patterns were identified: (i) the 'condiments' pattern; (ii) the 'animal and plant protein' pattern; (iii) the 'healthy traditional' pattern; (iv) the 'fruits, eggs and juice' pattern; and (v) the 'alcohol, milk and tea' pattern. A total of $2093(72 \cdot 2 \%)$ individuals completed the follow-up survey and the 3 -year cumulative incidence of hyperglycaemia was $7 \cdot 5 \%$ (158/2093). A 1-unit increase in the score for the 'healthy traditional' pattern was associated with a decrease of $0.054 \mathrm{mmol} / \mathrm{l}$ in fasting plasma glucose $(P=0.017)$, while a 1-unit increase in the 'fruits, eggs and juice' pattern score was associated with an increase of 0.050 $\mathrm{mmol} / \mathrm{l}$ in fasting plasma glucose $(P=0.023)$ by multivariable linear regression. For men, tertile 3 of the 'fruits, eggs and juice' pattern was associated with an $88 \%$ greater risk (hazard ratio $=1 \cdot 88 ; 95 \%$ CI 1.04, 3.54) of hyperglycaemia than tertile 1 of this pattern. Being in tertile 3 of the 'alcohol, milk and tea' pattern was associated with a $35 \%$ greater risk (hazard ratio $=1 \cdot 35 ; 95 \%$ CI $1 \cdot 04,2 \cdot 16$ ) relative to tertile 1 in women, while for the 'healthy traditional' pattern tertile 3 was associated with a $41 \%$ lower risk (hazard ratio $=0.59 ; 95 \%$ CI $0.35,0.99$ ) compared with tertile 1 . The 'condiments' and the 'animal and plant protein' patterns were not independently associated with hyperglycaemia.

Conclusions: Our findings suggest that modifying dietary patterns could reduce hyperglycaemia incidence in the mainland Chinese adult population.
\end{abstract}

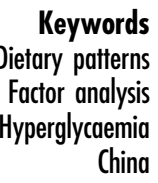

In the past two decades, there has been an overall increase of diabetes mellitus prevalence in developing countries $^{(1,2)}$. According to the International Diabetes Federation, approximately 366 million adults were diagnosed with diabetes in 2011 and 552 million adults are expected to be affected by diabetes by $2030^{(3)}$. A national survey conducted in 2010 showed that the weighted prevalence of type 2 diabetes (T2D) was $9.7 \%$ among Chinese adults aged 18 years and above ${ }^{(4)}$. The ageing of the population, urbanization, obesity, physical inactivity 
and unhealthful diets have probably contributed to the rapid increase in the diabetes burden in China ${ }^{(5,6)}$. Thus, prevention of T2D through lifestyle factors should be a high public health priority.

It is well accepted that nutritional factors play an important role in the aetiology of T2D. Traditional analyses typically examine T2D in relation to a single or a few nutrients or food items ${ }^{(7)}$. However, this has several conceptual and methodological limitations ${ }^{(8)}$. First, people do not eat isolated nutrients. Instead, they eat meals consisting of a variety of foods with complex combinations of nutrients. Also, collinearity among some nutrients has made it difficult to separate out their individual effects. Third, the effect of a single nutrient may be too small to detect.

Dietary pattern analysis has been used increasingly as an alternative method to traditional analysis because it takes into account the diet's overall effects ${ }^{(8)}$. Three main approaches have been used to define dietary patterns: factor analysis, cluster analysis and dietary indices. Factor analysis has reasonable reproducibility and validity, and is a data reduction technique that reduces the complexity of diet to a small number of dimensions, based on the intercorrelations between food items ${ }^{(8-10)}$.

Epidemiological studies have examined associations between dietary patterns and the risk of T2D ${ }^{(11-21)}$. Generally, these studies suggest that the pattern including higher intakes of vegetables and fruits, whole grains, fish and low-fat dairy may decrease diabetes risk, and the pattern including higher intakes of processed grains, sugars, processed and red meats, and fried foods may increase diabetes risk. Most of these studies have focused on foreign countries ${ }^{(11-14,17-21)}$. Information on dietary patterns and the risk of hyperglycaemia in Chinese population is scarce ${ }^{(15,16)}$.

Accordingly, the aims of the present study were to identify dietary patterns by using exploratory factor analysis and to examine the association of dietary patterns with the risk of developing hyperglycaemia after 3 years of follow-up in a community-based cohort of the mainland Chinese adult population.

\section{Methods}

\section{Study sample and design}

The community-based prospective cohort study was conducted between 2007 and 2010 in urban areas of Nanjing, one of the largest cities in east mainland China. In 2007, Nanjing had a registered population of more than 6.1 million with eleven urban districts and two rural counties. A multistage random sampling method was adopted. First, we randomly selected two urban districts. Then, three or four streets in each chosen district were randomly selected. Finally, one community in each chosen street was randomly selected. This resulted in a total number of seven communities. All eligible residents within each selected community were invited to participate in the baseline survey from June to September 2007. The inclusion criteria were local resident for at least 5 years, aged 30 years or above, free of hyperglycaemia and other serious diseases requiring a special diet, and not on a weight-reduction diet in 2007. After exclusions, from a total of eligible 3376 individuals, 2900 agreed to participate in the baseline survey (response rate $=85.9 \%$ ) and were invited to re-attend the follow-up survey from June to September 2010. Of these participants, 2093 (873 male and 1220 female) completed the follow-up survey (follow-up rate $=72.2 \%$ ). The reasons for attrition were refusal, absence during the investigation period, poor health or other reasons. No significant differences existed between participants who were followed up and lost to follow-up in terms of age, education, occupation, income, cigarette smoking, alcohol intake, physical activity, BMI and food intake at baseline, except gender (data not shown). Written informed consent was obtained from each participant prior to the survey. The study was approved by the academic and ethical committee of Nanjing Municipal Center for Disease Control and Prevention (Nanjing CDC).

\section{Investigated measures}

The tools for data collection included laboratory measures, anthropometric measures and interviewer-assisted questionnaire surveys. Participants completed a face-to-face interview administered by trained health-care professionals. A structured questionnaire was designed to collect information on diet, sociodemographic information (age, sex, education, occupation and income), medical history, lifestyle and behavioural factors (cigarette smoking, alcohol intake and physical activity).

\section{Laboratory measures}

We collected a blood sample from each participant to assess fasting plasma glucose (FPG) concentration at the baseline survey and then excluded those with hyperglycaemia at baseline. We collected another blood sample from each cohort member at the 3-year follow-up survey to identify the new occurrence of hyperglycaemia.

For this purpose, after at least $10 \mathrm{~h}$ of overnight fasting, a venous blood sample was collected from each participant into a vacuum tube containing sodium fluoride for the measurement of FPG at both baseline and 3-year follow-up surveys. FPG was measured by the glucose oxidase method in a designated local hospital.

\section{Outcome variable (definition of byperglycaemia)}

Hyperglycaemia was defined as FPG concentration of $\geq 6.1 \mathrm{mmol} / 1$ or already taking oral hyperglycaemia agents for treatment of $\mathrm{T} 2 \mathrm{D}^{(22,23)}$

\section{Anthropometric measures}

Anthropometric measures including weight, height and waist circumference (WC) were taken at baseline recruitment 
according to a standard protocol by trained interviewers. Height was measured without shoes to the nearest $0 \cdot 1 \mathrm{~cm}$ and weight was measured with light clothing to the nearest $0.1 \mathrm{~kg}$. WC was measured to the nearest $0.1 \mathrm{~cm}$ midway between the inferior margin of the last rib and the iliac crest in a horizontal plane. BMI was calculated as weight in kilograms divided by the square of height in metres. General obesity was defined as BMI $\geq 28.0 \mathrm{~kg} / \mathrm{m}^{2}$, overweight was defined as $24.0 \leq \mathrm{BMI}<28.0 \mathrm{~kg} / \mathrm{m}^{2}$, normal weight was defined as $18.5 \leq \mathrm{BMI}<24.0 \mathrm{~kg} / \mathrm{m}^{2}$ and underweight was defined as BMI $<18.5 \mathrm{~kg} / \mathrm{m}^{2}$ according to the Chinese standard ${ }^{(24)}$.

\section{Covariates}

Socio-economic status

Socio-economic status (SES) was assessed by means of three variables. Education was recoded into three categories: (i) primary school and lower; (ii) middle/high school; and (iii) college and higher. Occupation was grouped into: (i) manual labourers (farmer, factory worker, forestry worker, fisherman); (ii) service staff (salesperson, house worker); and (iii) mental labourers (office worker, teacher, doctor and retired person) ${ }^{(25)}$. The total annual income of all family members was divided into tertiles to derive three categories: (i) lower; (ii) middle; and (iii) higher ${ }^{(26)}$.

\section{Cigarette smoking}

The categories of smoking status were defined as: (i) current smokers (those who smoked at least one cigarette daily continuously for at least 1 year, or smoked at least eighteen packs in total each year); (ii) ex-smokers (those who previously smoked but subsequently quit); and (iii) non-smokers (those who never smoked cigarettes) ${ }^{(27)}$. Both current smokers and ex-smokers were categorized as smokers in the present study.

\section{Alcohol intake}

The categories of drinking status were defined as: (i) alcohol drinkers (those who drank alcohol at least once weekly for at least 1 year); and (ii) non-drinkers (those who never drank or did not meet the criterion for alcohol drinkers) ${ }^{(28)}$.

\section{Physical activity}

Physical activity was assessed using the validated Chinese version of the International Physical Activity Questionnaire $(\text { IPAQ })^{(29)}$. Participants were categorized as: (i) yes (had $\geq 150 \mathrm{~min} /$ week) and (ii) no ( $<150 \mathrm{~min} /$ week), according to the time they were engaged in moderate-intensity physical activity.

\section{Dietary assessment}

Dietary intake during the previous year was assessed at baseline via an interviewer-administered FFQ, the reliability and validity of which have been examined elsewhere ${ }^{(30)}$. Test-retest reliability was assessed in twenty-one individuals who had repeated administrations of the questionnaire $14 \mathrm{~d}$ apart (FFQ1, FFQ2). The validity of the FFQ was assessed by comparing nutrient intakes with those from a $4 \mathrm{~d}$ food record. Pearson correlation coefficients and intra-class correlation coefficients for reliability of the FFQ ranged from 0.51 to 0.85 and from 0.51 to 0.81 , respectively. Correlation coefficients and intra-class correlation coefficients for validity of the FFQ ranged from 0.36 to 0.69 and from 0.34 to 0.57 , respectively.

The FFQ included eighty-seven food items and twelve food categories: (i) grains and products; (ii) red meat (pork, beef/lamb); (iii) poultry meat; (iv) fish and shrimp; (v) eggs; (vi) dairy products; (vii) soya foods; (viii) vegetables; (ix) fruits; (x) beverages; (xi) snacks/desserts; and (xii) condiments, which covered $90 \%$ of the commonly consumed foods in urban Nanjing. Participants were asked to recall the frequency of consumption of each food item (daily, weekly, monthly, annually, never) and the estimated portion size, using a common unit of weight in China (1 liang $=50 \mathrm{~g}$ ) or natural units (cups). Intakes of foods were converted into $\mathrm{g} / \mathrm{d}$ (the number of liangs were translated to grams by multiplying by 50 ) for data analysis. To reduce the complexity of the data and the low intake of some food items, food items were aggregated into groups. We formed twenty-eight separate food groups on the basis of similarity of type of food and nutrient composition ${ }^{(31,32)}$ (see Appendix).

Dietary patterns (main independent variables) were identified from twenty-eight food groups by exploratory factor analysis, using the standard principal component analysis method. Factors were rotated by orthogonal transformation (varimax rotation) to improve interpretability and minimize the correlation between the factors. The number of factors retained was based on the eigenvalue $(>1 \cdot 0)$, scree plot, factor interpretability and the variance explained by each factor ${ }^{(33,34)}$. Factor loadings represented correlation coefficients between food groups and dietary patterns. Food groups with positive loadings contributed to the dietary pattern, whereas those with negative loadings were inversely associated with the dietary pattern. Food groups with absolute factor loadings $\geq 0.20$ were considered as contributing significantly to the pattern ${ }^{(35)}$. The dietary patterns were named according to the food items showing high loadings (absolute value) in each dietary pattern. The proportion of variance explained by each factor was calculated by dividing the sum of the squares of the respective factor loadings by the number of variables. Dietary factor scores for each participant were calculated by summing the standardized intakes of each food group weighted by the factor loading ${ }^{(35)}$.

\section{Statistical analysis}

Before performing principal component analysis, data suitability for factor analysis was assessed using the Kaiser-Meyer-Olkin (KMO) measure of sampling adequacy $(>0 \cdot 6)$ and Bartlett's test of sphericity $(<0 \cdot 05)$. 
Participants were categorized into tertiles of dietary factor score for each dietary pattern, and participants in the lowest tertile were chosen as the reference group. Means and standard deviations for age, BMI, WC and FPG were calculated according to tertiles of dietary pattern scores using one-way ANOVA. Associations between dietary patterns and sociodemographic factors were investigated using $\chi^{2}$ analysis. Multivariable linear modelling was used to analyse the association between dietary pattern factor scores and FPG after 3-year follow-up. Multivariable Cox proportional hazards regression ${ }^{(18-21,23)}$ was used to estimate hyperglycaemia incidence hazard ratios (HR) comparing each tertile with the lowest. All regression models were controlled for age, sex, SES, cigarette smoking, alcohol intake, physical activity, total energy intake and BMI at baseline. All analyses were performed using the statistical software package IBM SPSS Statistics 20.0. A value of $P<0.05$ was considered statistically significant.

\section{Results}

\section{Dietary patterns}

Favourable results $(\mathrm{KMO}=0.702$, Barlett's $P<0.001)$ supporting the factor ability of the correlation matrix were obtained. Five dietary patterns were extracted from twenty-eight food groups in the FFQ using factor analysis. The factor loading matrices are shown in Table 1. Factor 1, which loaded heavily on soya sauce, sugar, vinegar and salt, was labelled the 'condiments' pattern. Factor 2, with high loadings for poultry meat, dry beans, other soyabean products and livestock meat, was labelled the 'animal and plant protein' pattern. Factor 3, with high loadings for green vegetables, rice and products, dry vegetables, other vegetables, and fish and shrimp, was labelled the 'healthy traditional' pattern. Factor 4, with high loadings for fruits, eggs, soyabean milk, juice, nuts, cola and sweets, was labelled the 'fruits, eggs and juice' pattern. Factor 5, with high loadings for beer, liquor, dairy products, wine and tea, was labelled the 'alcohol, milk and tea' pattern. The eigenvalue was 2.63, 2.39, 1.66, 1.39 and 1.25 for each factor, respectively. Overall, the five dietary patterns explained $33.2 \%$ of the variance in food intakes $(8.9 \%, 7.5 \%, 6.3 \%, 5.9 \%$ and $4.6 \%$ for factors $1-5$, respectively).

\section{Characteristics of study participants by dietary patterns}

Table 2 demonstrates associations of dietary patterns with sociodemographic and anthropometric characteristics at baseline. Age differed significantly across tertiles of each dietary pattern. Participants with a high level of the 'traditional health' pattern had lower BMI, WC and FPG than those with a low level of this dietary pattern. Conversely, the 'fruits, eggs and juice' pattern was positively associated with FPG and the 'alcohol, milk and tea' pattern was positively

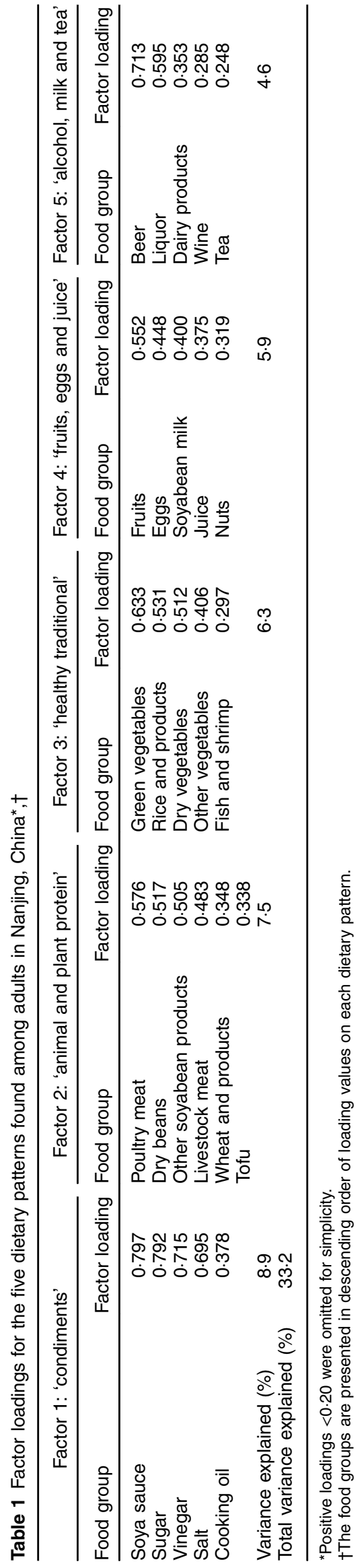




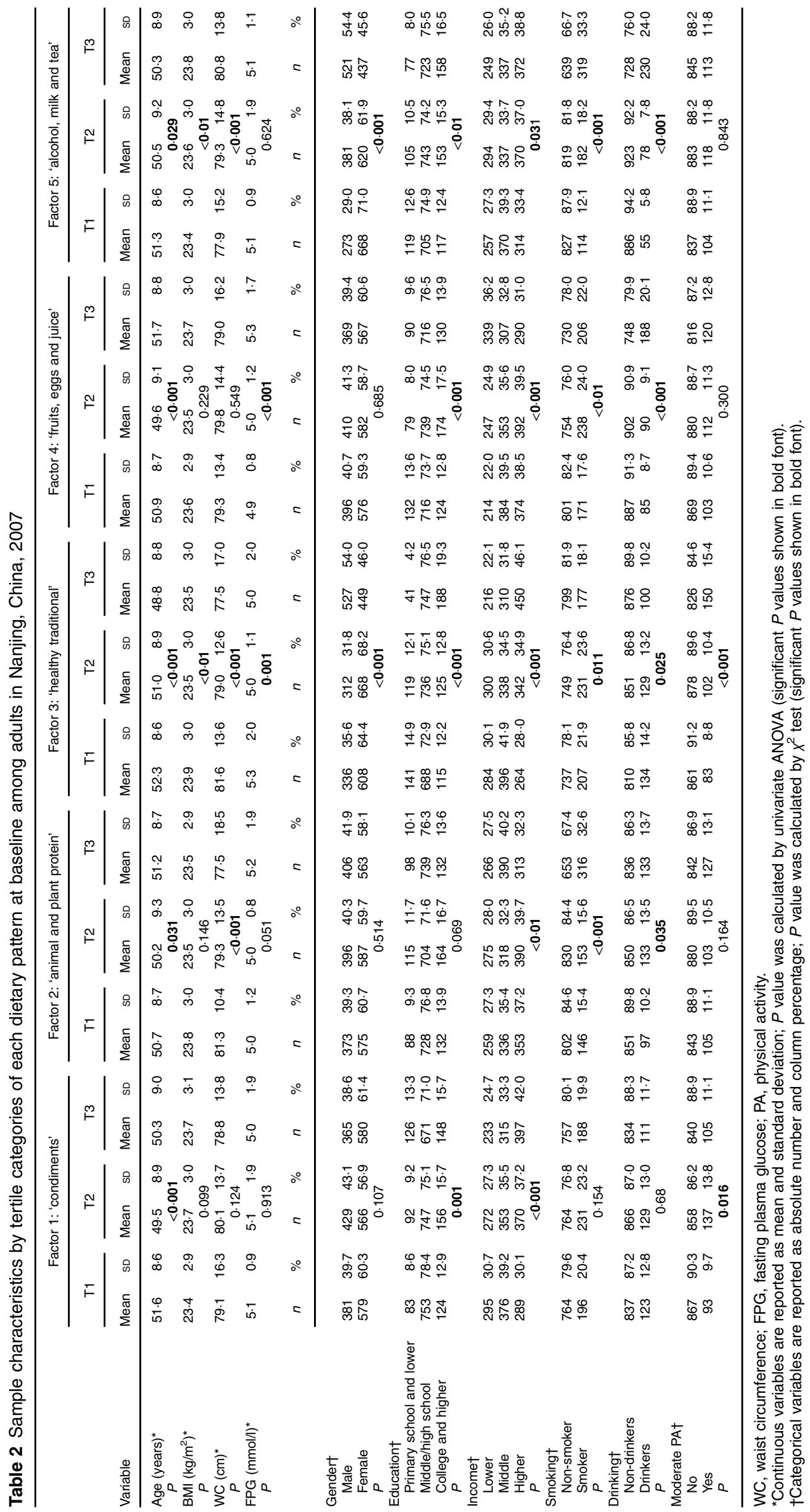


Table 3 Multivariate-adjusted linear associations between each dietary pattern and fasting plasma glucose at baseline among adults in Nanjing, China, 2007

\begin{tabular}{|c|c|c|c|c|c|c|}
\hline \multirow[b]{2}{*}{ Dietary pattern } & \multicolumn{2}{|c|}{ Total } & \multicolumn{2}{|c|}{ Males } & \multicolumn{2}{|c|}{ Females } \\
\hline & $\operatorname{Std} \beta$ & $P$ & $\operatorname{Std} \beta$ & $P$ & Std $\beta$ & $P$ \\
\hline 'Condiments' & -0.023 & 0.290 & 0.006 & 0.865 & -0.032 & 0.266 \\
\hline 'Animal and plant protein' & 0.012 & 0.595 & 0.070 & 0.036 & -0.001 & 0.972 \\
\hline 'Healthy traditional' & -0.054 & 0.017 & -0.124 & 0.000 & -0.034 & 0.249 \\
\hline 'Fruits, eggs and juice' & 0.050 & 0.023 & 0.079 & 0.019 & 0.041 & 0.152 \\
\hline 'Alcohol, milk and tea' & 0.024 & 0.302 & 0.047 & 0.171 & 0.027 & 0.355 \\
\hline
\end{tabular}

Multivariate linear model adjusted for age, sex, socio-economic status, cigarette smoking, alcohol intake, physical activity, total energy intake and BMI at baseline (significant associations shown in bold font).

associated with BMI and WC. The 'condiments' pattern was more representative in those with lower education level and those who were less physically active, but with higher income. People with a higher score for the 'animal and plant protein' and the 'fruits, eggs and juice' patterns had lower income, but were more likely to smoke cigarettes and drink alcohol than people with a lower score. Compared with participants in the lowest tertile, those in the highest tertile of the 'health traditional' pattern were more likely to be male, have a high education level, higher income and be more physically active, but were less likely to smoke and drink alcohol. Those in the highest tertile of the 'alcohol, milk and tea' dietary pattern tended to be male, have high SES, smoke and drink more than those in the lowest tertile.

\section{Relationship between dietary pattern scores and byperglycaemia risk after 3-year follow-up}

A 1-unit increase in the score for the 'healthy traditional' pattern was associated with a decrease of $0.054 \mathrm{mmol} / \mathrm{l}$ in FPG $(P=0.017)$, while a 1-unit increase in 'fruits, eggs and juice' pattern score was associated with an increase of $0.050 \mathrm{mmol} / 1$ in FPG $(P=0.023)$ by multivariable linear regression (Table 3 ).

Three-year cumulative incidence of hyperglycaemia was $7.5 \%$ (158/2093). The incidence rate was $7 \cdot 1 \%$ (62/873) for males and $7.9 \%(96 / 1220)$ for females, but the differences were not statistically significant $\left(\chi^{2}=0.43\right.$, $P=0.513$ ). By multivariable Cox regression analysis (with adjustment for age, sex, SES, cigarette smoking, alcohol intake, physical activity, total energy intake and BMI at baseline), for men, tertile 3 of the 'fruits, eggs and juice' pattern was associated with an $88 \%$ greater risk (HR $=1.88 ; 95 \%$ CI $1.04,3.54)$ of hyperglycaemia than tertile 1 of this pattern. Being in tertile 3 of the 'alcohol, milk and tea' pattern was associated with a $35 \%$ greater risk $(\mathrm{HR}=1 \cdot 35 ; 95 \% \mathrm{CI} 1 \cdot 04,2 \cdot 16)$ relative to tertile 1 in women, while for the 'healthy traditional' pattern tertile 3 was associated with a $41 \%$ lower risk ( $\mathrm{HR}=0.59$; $95 \%$ CI $0.35,0.99)$ than tertile 1 . However, no association was noted in the occurrence of hyperglycaemia with the 'condiments' and the 'animal and plant protein' patterns (Table 4).

\section{Discussion}

In the current prospective study of the Nanjing Chinese adult population, five dietary patterns were identified using factor analysis: (i) the 'condiments' pattern; (ii) the 'animal and plant protein' pattern; (iii) the 'healthy traditional' pattern; (iv) the 'fruits, eggs and juice' pattern; and (v) the 'alcohol, milk and tea' pattern. The 'healthy traditional' pattern, rich in vegetables, rice and products, fish and shrimp, was associated with a lower risk of hyperglycaemia. In contrast, the pattern 'fruits, eggs and juice' and the pattern 'alcohol, milk and tea' were associated with a significantly higher risk of hyperglycaemia in Chinese men and women in Nanjing. These associations were independent of age, sex, SES, cigarette smoking, alcohol intake, physical activity, total energy intake and BMI at baseline. However, no significant associations were observed between the 'condiments' and the 'animal and plant protein' patterns and the development of hyperglycaemia.

In contrast to the study of individual nutrients or foods, dietary pattern analysis considers the overall diet, reflecting more closely real-world habits. Dietary patterns derived by factor analysis can be proxy indicators of the real food consumption and availability conditions of a population, providing a more realistic representation of the everyday eating habits ${ }^{(8)}$.

The patterns identified in the present study are similar to previous findings in China. Two major dietary patterns were identified, (i) a vegetable, fruit and soya-rich pattern (VFS) and (ii) a dim sum, meat-rich pattern (DSM), from the Singapore Chinese Health Study ${ }^{(11)}$. Four major dietary patterns were reported in the Shanghai Women's Study ${ }^{(36)}$ : the first pattern was characterized by higher intakes of eggs, legumes and products; the second pattern was characterized by higher intakes of oil, sugar and condiments; the third pattern was characterized by higher intakes of dairy, vegetables and rice; while the fourth pattern was characterized by higher intakes of fruits, beef and fish. In Jiangyin ${ }^{(37)}$, five dietary patterns were identified: (i) a 'thrifty' pattern (rich in dry beans, other soyabean products and whole grains); (ii) 'healthy' pattern (rich in vegetables, fish, poultry meat and livestock meat); (iii) 'sweet food' pattern (rich in dairy products, soyabean 


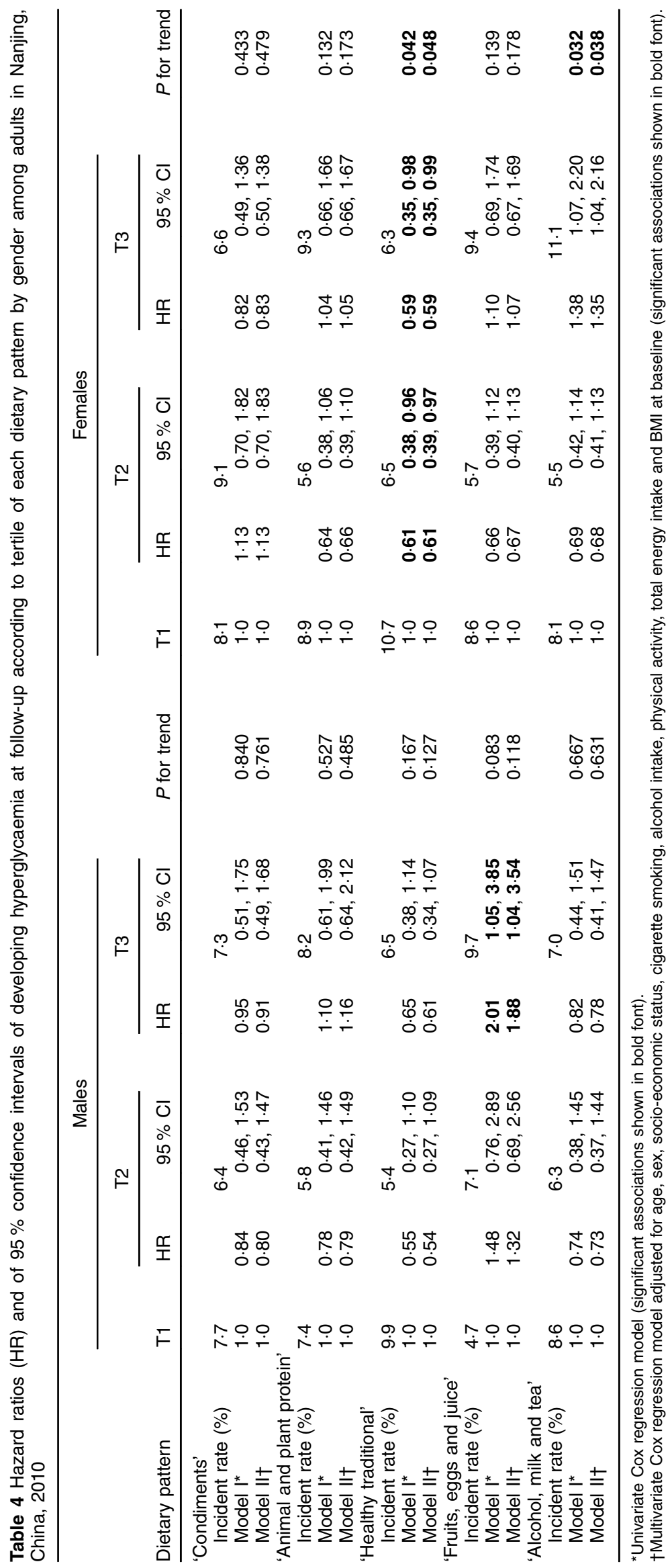


milk, sweets and desserts); (iv) a 'beverage' pattern (rich in juice and cola); and (v) 'drinking' pattern (rich in beer, liquor and wine).

Several prospective studies have evaluated the relationship of dietary patterns with incidence of T2D in Western populations ${ }^{(11-14,17-21)}$, but data from Chinese populations are scarce ${ }^{(15,16)}$. Many authors have described two major patterns ${ }^{(13,14,17-19)}$ : one labelled 'healthy' or 'prudent' usually with vegetables, fruits, legumes, whole grains, low-fat dairy products, poultry and fish that was associated with reduced risk of diabetes, whereas the other labelled 'unhealthy' or 'western' with processed meat, red meat, butter, high-fat dairy products, eggs, snacks, sweets/desserts and refined grains was associated with increased risk. It is hardly new that moving from the 'unhealthy' pattern to the healthier pattern could reduce diabetes incidence.

In the present study, the 'healthy traditional' pattern (high in vegetables, rice and products, fish and shrimp) was the traditional Chinese diet, with a low fat content and plenty of vegetables. The negative association of hyperglycaemia and the 'healthy traditional' pattern in men is in agreement with similar patterns in several other cohort studies $^{(13,17-19)}$ and a recent meta-analysis ${ }^{(38)}$ that associated vegetables, whole grains and fish with lower incidence of T2D. In the Nurses' Health Study ${ }^{(17)}$, the Health Professionals Follow-up Study ${ }^{(18)}$ and the Finnish Mobile Clinic Health Examination Survey ${ }^{(13)}$, inverse associations were found between the 'prudent' dietary pattern and T2D. A dietary pattern with higher intake of vegetables, fruits and soya foods was inversely associated with risk of incident T2D in the Singapore Chinese Health Study $^{(11)}$. In the follow-up study of Hong Kong Chinese adults $^{(15)}$, the more vegetables, fruit and fish pattern was associated with a lower risk of diabetes. These dietary habits associated with reduced diabetes incidence may be considered as anti-inflammatory and antioxidative, principally because of the increased intake of protective nutrients such as dietary fibre, vitamins and minerals, as well as antioxidants and polyphenols ${ }^{(38)}$.

In the past two decades, China has experienced a significant nutrition transition from the traditional Chinese diet to a Western pattern diet, with an increase in consumption of red meats, eggs and oils, and a decrease in fruit and vegetable intake ${ }^{(39)}$. In the present study, the positive associations for the 'fruits, eggs and juice' pattern in men and the 'alcohol, milk and tea' pattern in women are consistent with studies that showed an increased risk of hyperglycaemia for persons adhering to a Western or unhealthy diet. In an urban Mexican population ${ }^{(40)}$ it was shown that consumption of a Western pattern was associated with $71 \%$ increase in the risk of having high FPG. Lebanese adults ${ }^{(41)}$ belonging to the fifth quintile of the 'fast food/dessert' factor score (rich in fast foods, sandwiches including hamburgers, chawarma and falafel in addition to pizzas, pies, desserts, carbonated beverages, juices and mayonnaise) had significantly higher odds of hyperglycaemia. In Sweden ${ }^{(42)}$, the most striking findings were a higher risk of hyperglycaemia with the 'many foods and drinks' pattern (with high intakes of cheese, cake, fat meat and alcohol) for men. The positive effects of the 'fruits, eggs and juice' and 'alcohol, milk and tea' patterns can be attributed to their higher saturated fat and carbohydrate content and excess energy intake, which may lead to hyperglycaemia ${ }^{(38)}$. A very high intake of fruits and especially juice might be unbeneficial for the Nanjing Chinese population, as other researchers ${ }^{(20,43)}$ also have found. Dairy products have been associated with diabetes risk due to their high fat content, but low-fat dairy products may have beneficial effects ${ }^{(21,44)}$. Unfortunately, we were not able to differentiate between high- and low-fat products. Consistent with our results, many epidemiological studies ${ }^{(45,46)}$ have reported a positive association between alcohol consumption and diabetes risk.

The inconsistent results for the relationship between dietary patterns and risk hyperglycaemia by sex may be due to diverse dietary habits observed in men and women. Men had higher scores on the 'healthy traditional' and the 'alcohol, milk and tea' patterns and relatively higher intakes of vegetables, alcohol consumption and dairy products.

Previous studies ${ }^{(41,47)}$ have reported that dietary patterns were related to sociodemographic characteristics and lifestyle behaviours. Higher SES (more likely to have higher income and education levels) was usually found to be associated with higher scores on the healthy dietary pattern $^{(48)}$. Unhealthy lifestyle behaviours such as cigarette smoking, alcohol intake and physical inactivity were reportedly related to unhealthy dietary patterns ${ }^{(13,47)}$. Our results were in accordance with these studies. In the present study, male, high education level, higher income, more physically active, non-smoker and non-drinker were more likely among those adhering to the 'healthy traditional' pattern. In contrast, people with a higher score for the 'animal and plant protein' and the 'fruits, eggs and juice' patterns had lower income, but were more likely to smoke cigarettes and drink alcohol than people with a lower score. The relationship between higher SES and the 'healthy traditional' pattern could be because of increased knowledge and health awareness, or increased pressures of social acceptability that occur with increasing SES, which may influence people's food consumption habits. Among the lower SES groups, the increased cost of healthier foods or decreased access to healthier foods may be important factors influencing their food choices. People who have less money could choose to buy cheaper foods and these cheaper foods are generally unhealthy ${ }^{(48)}$.

The present study had several potential limitations. First, compared with relevant foreign studies ${ }^{(11-14,17-21)}$, the sample size was small and the duration of follow-up period was short, so some predisposing factors may not have achieved statistical significance. Moreover, the complete 
follow-up rate was relatively low. Although no significant differences existed between participants who were followed up and those lost to follow-up, the bias caused by these missing data should be taken into consideration. Second, dietary intake was assessed by FFQ. The major sources of errors in FFQ include memory, interpretation of questions, perception of portion sizes and the restricted food list. However, FFQ have become commonly used instruments for measuring relationships between nutrient intakes and diseases in epidemiological studies in Western countries in recent decades. Third, dietary patterns are likely to vary according to sex, age, area of residence, ethnic group and culture. It is possible that different eating patterns are derived from different populations ${ }^{(8,16,34)}$. This does not necessarily refute the validity of pattern analy$\mathrm{sis}^{(8,31,34,49)}$. Furthermore, decisions based on factor analysis (including the consolidation of food items into food groups, the number of factors to extract, the method of rotation and the labelling of the components) may be subjective or arbitrary and can affect the study results and interpretation $^{(50)}$. Factor analysis is considered an a posteriori approach because it generates patterns based on available empirical data without a priori hypotheses. Nevertheless, this method offers an opportunity to summarize and refine large amounts of data into a simple descriptive pattern ${ }^{(8-10)}$. Finally, we used BMI instead of WC for an obesity index. However, BMI is widely accepted and has consistently been shown to be related to diabetes risk factors in the Chinese population ${ }^{(51)}$.

The present study has several strengths. First, the population-based prospective study design can minimize selection bias and examine the causal relationship between dietary patterns and the risk of hyperglycaemia. Second, the comprehensive information on lifestyle and dietary factors allowed for adjustment of a broad range of potential confounding variables. Finally, we collected individual food consumption information by an interviewer-administered FFQ. Based on the current educational levels of most of the Chinese population, a self-administered FFQ survey is not advisable and a face-to-face interview is the proper approach $^{(33)}$. In addition, the experienced interviewer is able to obtain more precise and accurate estimates of the different portion sizes of each food consumed, thus minimizing the error involved in having participants try to estimate the amount of food consumed and the between-person error in portion size estimation among participants ${ }^{(30,52)}$.

\section{Conclusion}

Our results indicated that a dietary pattern characterized by higher intakes of green vegetables, rice and products, dry vegetables, other vegetables, and fish and shrimp was inversely associated with risk of incident hyperglycaemia, whereas a pattern with higher intakes of fruits, soyabean milk, juice, nuts, cola and sweets and a pattern with higher intakes of beer, liquor, dairy products, wine and tea were associated with a significantly increased risk of hyperglycaemia in a cohort of Chinese men and women in Nanjing. Dietary patterns might be important in the prevention of hyperglycaemia in Chinese adult populations.

\section{Acknowledgements}

Acknowledgements: The authors are grateful to all the dedicated fieldworkers who took part in the surveys and all participants who facilitated the survey implementation at each community. Financial support: The study was supported by Nanjing Municipal Medical Science and Technique Development Foundation, China (grant number 2007-ZKX07026). Nanjing Municipal Medical Science and Technique Development Foundation had no role in the design, analysis or writing of this article. Conflict of interest: None. Authorship: X.H. contributed to data analysis and manuscript writing; F.X. (Principal Investigator of the project) was responsible for study design, data collection and analysis, and manuscript writing; Z.W. and Y.L. were responsible for data collection and manuscript revision; J.L. (Co-Principal Investigator of the project) was responsible for study design, data collection and manuscript writing. All authors read and approved the final manuscript. Ethics of buman subject participation: This study was approved by the academic and ethical committee of Nanjing Municipal Center for Disease Control and Prevention (Nanjing CDC). Written informed consent was obtained from each participant prior to the survey.

\section{References}

1. Rathmann W \& Giani G (2004) Global prevalence of diabetes: estimates for the year 2000 and projections for 2030. Diabetes Care 27, 2568-2569.

2. Yang G, Kong L, Zhao W et al. (2008) Emergence of chronic non-communicable diseases in China. Lancet 372, 1697-1705.

3. Whiting DR, Guariguata L, Weil C et al. (2011) IDF diabetes atlas: global estimates of the prevalence of diabetes for 2011 and 2030. Diabetes Res Clin Pract 94, 311-321.

4. National Center for Chronic and Non-communicable Disease Control and Prevention, Chinese Center for Disease Control and Prevention (2010) Report on Chronic Disease Risk Factor Surveillance in China. Beijing: Military Medical Science Press (in Chinese).

5. Li MZ, Su L, Liang BY et al. (2013) Trends in prevalence, awareness, treatment, and control of diabetes mellitus in mainland china from 1979 to 2012. Int J Endocrinol 2013 , 753150 .

6. Zuo H, Shi Z \& Hussain A (2014) Prevalence, trends and risk factors for the diabetes epidemic in China: a systematic review and meta-analysis. Diabetes Res Clin Pract 104, 63-72.

7. Virtanen SM \& Aro A (1994) Dietary factors in the aetiology of diabetes. Ann Med 26, 469-478.

8. Hu FB (2002) Dietary pattern analysis: a new direction in nutritional epidemiology. Curr Opin Lipidol 13, 3-9.

9. DiBello JR, Kraft P, McGarvey ST et al. (2008) Comparison of 3 methods for identifying dietary patterns associated with risk of disease. Am J Epidemiol 168, 1433-1443.

10. Reedy J, Wirfält E, Flood A et al. (2010) Comparing 3 dietary pattern methods - cluster analysis, factor analysis, and index 
analysis - with colorectal cancer risk: the NIH-AARP Diet and Health Study. Am J Epidemiol 171, 479-487.

11. Odegaard AO, Koh WP, Butler LM et al. (2011) Dietary patterns and incident type 2 diabetes in Chinese men and women: The Singapore Chinese Health Study. Diabetes Care 34, 880-885.

12. Erber E, Hopping BN, Grandinetti A et al. (2010) Dietary patterns and risk for diabetes: the multiethnic cohort. Diabetes Care 33, 532-538.

13. Montonen J, Knekt P, Härkänen T et al. (2005) Dietary patterns and the incidence of type 2 diabetes. Am J Epidemiol 161, 219-227.

14. Malik VS, Fung TT, van Dam RM et al. (2012) Dietary patterns during adolescence and risk of type 2 diabetes in middle-aged women. Diabetes Care 35, 12-18.

15. Yu R, Woo J, Chan R et al. (2011) Relationship between dietary intake and the development of type 2 diabetes in a Chinese population: the Hong Kong Dietary Survey. Public Health Nutr 14, 1133-1141.

16. Villegas R, Yang G, Gao YT et al. (2010) Dietary patterns are associated with lower incidence of type 2 diabetes in middle-aged women: the Shanghai Women's Health Study. Int J Epidemiol 39, 889-899.

17. Fung TT, Schulze M, Manson JE et al. (2004) Dietary patterns, meat intake, and the risk of type 2 diabetes in women. Arch Intern Med 164, 2235-2240.

18. van Dam RM, Rimm EB, Willett WC et al. (2002) Dietary patterns and risk for type 2 diabetes mellitus in US men. Ann Intern Med 136, 201-209.

19. McNaughton SA, Mishra GD \& Brunner EJ (2008) Dietary patterns, insulin resistance, and incidence of type 2 diabetes in the Whitehall II Study. Diabetes Care 31, 1343-1348.

20. Hodge AM, English DR, O'Dea K et al. (2007) Dietary patterns and diabetes incidence in the Melbourne Collaborative Cohort Study. Am J Epidemiol 165, 603-610.

21. Nettleton JA, Steffen LM, Ni H et al. (2008) Dietary patterns and risk of incident type 2 diabetes in the Multi-Ethnic Study of Atherosclerosis (MESA). Diabetes Care 31, 1777-1782.

22. World Health Organization (1999) Definition, Diagnosis and Classification of Diabetes Mellitus and its Complications. Report of a WHO Consultation. Part I: Diagnosis and Classification of Diabetes Mellitus. Geneva: WHO.

23. The Expert Committee on the Diagnosis and Classification of Diabetes Mellitus (2003) Follow-up report on the diagnosis of diabetes mellitus. Diabetes Care 26, 3160-3167.

24. Zhou BF (2002) Predictive values of body mass index and waist circumference for risk factors of certain related diseases in Chinese adults: study on optimal cut-off points of body mass index and waist circumference in Chinese adults. Biomed Environ Sci 15, 83-96.

25. Xu F, Yin XM \& Wang Y (2007) The association between amount of cigarettes smoked and overweight, central obesity among Chinese adults in Nanjing, China. Asia Pac J Clin Nutr 16, 240-247.

26. Xu F, Yin XM, Zhang M et al. (2005) Family average income and body mass index above the healthy weight range among urban and rural residents in regional mainland China. Public Health Nutr 8, 47-51.

27. Yang G, Fan L, Tan J et al. (1999) Smoking in China: findings of the 1996 National Prevalence Survey. JAMA 282, 1247-1253.

28. Zhuang M, Yuan Z, Lin L et al. (2012) Reproducibility and relative validity of a food frequency questionnaire developed for adults in Taizhou, China. PLoS One 7, e48341.

29. Qu NN \& Li KJ (2004) Study on the reliability and validity of international physical activity questionnaire. Chin J Epidemiol 25, 265-268 (in Chinese).

30. Xu L, J Dibley M \& D'Este C (2004) Reliability and validity of a food-frequency questionnaire for Chinese postmenopausal women. Public Health Nutr 7, 91-98.
31. Hu FB, Rimm E, Smith-Warner SA et al. (1999) Reproducibility and validity of dietary patterns assessed by a food frequency questionnaire. Am J Clin Nutr 69, 243-249.

32. Qin Y, Melse-Boonstra A, Yuan B et al. (2012) Zinc biofortification of rice in China: a simulation of zinc intake with different dietary patterns. Nutrients 4, 517-528.

33. Shi Z, Hu X, Yuan B et al. (2008) Vegetable-rich food pattern is related to obesity in China. Int J Obes (Lond) 32, 975-984.

34. Nanri A, Shimazu T, Ishihara J et al. (2012) Reproducibility and validity of dietary patterns assessed by a food frequency questionnaire used in the 5-year follow-up survey of the Japan Public Health Center-Based Prospective Study. J Epidemiol 22, 205-215.

35. Hatcher LA (1994) Step-By-Step Approach to Using SAS for Factor Analysis and Structural Equation Modeling. Cary, NC: SAS Institute.

36. Dai X, He P, Zhang YF et al. (2010) Dietary pattern of Shanghai community-based middle and aged women. J Hyg Res 39, 472-477 (in Chinese).

37. Luo YZ, Chen XW, Miu GZ et al. (2009) Association between hypertension and dietary patterns in residents of Jiangyin city. Chin Public Health 25, 314-316 (in Chinese).

38. Esposito K, Kastorini CM, Panagiotakos DB et al. (2010) Prevention of type 2 diabetes by dietary patterns: a systematic review of prospective studies and meta-analysis. Metab Syndr Relat Disord 8, 471-476.

39. Zhai F, Wang H, Du S et al. (2009) Prospective study on nutrition transition in China. Nutr Rev 67, Suppl. 1, S56-S61.

40. Denova-Gutiérrez E, Castañón S, Talavera JO et al. (2010) Dietary patterns are associated with metabolic syndrome in an urban Mexican population. J Nutr 140, 1855-1863.

41. Naja F, Nasreddine L, Itani L et al. (2013) Association between dietary patterns and the risk of metabolic syndrome among Lebanese adults. Eur J Nutr 52, 97-105.

42. Wirfält E, Hedblad B, Gullberg B et al. (2001) Food patterns and components of the metabolic syndrome in men and women: a cross-sectional study within the Malmö Diet and Cancer cohort. Am J Epidemiol 154, 1150-1159.

43. Villegas R, Shu XO, Gao YT et al. (2008) Vegetable but not fruit consumption reduces the risk of type 2 diabetes in Chinese women. J Nutr 138, 574-580.

44. Choi HK, Willett WC, Stampfer MJ et al. (2005) Dairyconsumption and risk of type 2 diabetes mellitus in men: a prospective study. Arch Intern Med 165, 997-1003.

45. Wakabayashi I (2014) Frequency of heavy alcohol drinking and risk of metabolic syndrome in middle-aged men. Alcohol Clin Exp Res 38, 1689-1696.

46. Rasouli B, Andersson T, Carlsson PO et al. (2014) Alcohol and the risk for latent autoimmune diabetes in adults: results based on Swedish ESTRID study. Eur J Endocrinol 171, 535-543.

47. Sun J, Buys N \& Shen S (2013) Dietary patterns and cardiovascular disease-related risks in Chinese older adults. Front Public Health 1, 48-56.

48. Mullie P, Clarys P, Hulens M et al. (2010) Dietary patterns and socioeconomic position. Eur J Clin Nutr 64, 231-238.

49. Khani BR, Ye W, Terry P et al. (2004) Reproducibility and validity of major dietary patterns among Swedish women assessed with a food-frequency questionnaire. J Nutr 134, $1541-1545$.

50. Martinez ME, Marshall JR \& Sechrest L (1998) Invited commentary: factor analysis and the search for objectivity. Am J Epidemiol 148, 17-19.

51. Ning F, Wang S, Wang Y et al. (2014) Weight change in association with the incidence of type 2 diabetes in adults from Qingdao, China. Zhong Hua Liu Xing Bing Xue Za Zbi 35, 764-768 (in Chinese).

52. Newby PK \& Tucker KL (2004) Empirically derived eating patterns using factor or cluster analysis: a review. Nutr Rev 62, $177-203$. 
Appendix

The twenty-eight food groups used in the dietary pattern analysis

\begin{tabular}{|c|c|}
\hline Food group & Food items \\
\hline 1. Rice and rice products & White rice, millet, porridge, rice flour \\
\hline 2. Wheat and products & Noodle, dumpling, bread, steamed bread, wonton, twist bread \\
\hline 3. Livestock meat & Beef, steak, lamb, pork, meatballs, meatloaf, ham \\
\hline 4. Poultry meat & Chicken, duck, goose \\
\hline 5. Fish and shrimp & Carp, grass carp, silver carp, herring, shrimp \\
\hline 6. Eggs & Egg \\
\hline 7. Dairy products & $\begin{array}{l}\text { Whole milk, low-fat milk, skimmed milk, yoghurt, cheese, cottage cheese, lactic acid bacteria-containing } \\
\text { beverages, ice cream }\end{array}$ \\
\hline 8. Tofu & Tofu \\
\hline 9. Soyabean milk & Soyabean milk \\
\hline 10. Dry beans & Red beans, green beans, black beans, soyabeans \\
\hline 11. Other soyabean products & Bean sprouts, soya chicken, bean curd sheet \\
\hline 12. Green vegetables & $\begin{array}{l}\text { Spinach, cucumber, Chinese cabbage, lettuce, broccoli, greens, celery, water spinach, } \\
\text { shepherd's purse }\end{array}$ \\
\hline 13. Other vegetables & Other than green vegetables \\
\hline 14. Fresh fruits & Oranges, bananas, apples, strawberries, grapes, peaches, pears, kiwifruit, melons, watermelon \\
\hline 15. Dry vegetables & Dried mushroom, dried black fungus, kelp, laver \\
\hline 16. Juice & Fruit or vegetable juice \\
\hline 17. Nuts & All nuts or seeds \\
\hline 18. Cola & Cola \\
\hline 19. Sweets & Candy, cookie, chocolate, honey, sugar \\
\hline 20. Tea & Red or green tea \\
\hline 21. Beer & Beer, regular or light \\
\hline 22. Liquor & Liquor \\
\hline 23. Wine & Red or white wine \\
\hline 24. Soya sauce & Soya sauce \\
\hline 25. Sugar & Sugar \\
\hline 26. Vinegar & Vinegar \\
\hline 27. Salt & Salt \\
\hline 28. Cooking oil & Cooking oil \\
\hline
\end{tabular}

